\title{
Trapezium-Type Inequalities for $k$-Fractional Integral via New Exponential-Type Convexity and Their Applications
}

\author{
Artion Kashuri $\mathbb{D}^{1},{ }^{1}$ Sajid Iqbal $\mathbb{D}^{2},{ }^{2}$ Saad Ihsan Butt $\mathbb{D}^{3},{ }^{3}$ Jamshed Nasir, ${ }^{4}$ \\ Kottakkaran Sooppy Nisar, ${ }^{5}$ and Thabet Abdeljawad $\mathbb{1}^{6,7,8}$ \\ ${ }^{1}$ Department of Mathematics, Faculty of Technical Science, University Ismail Qemali, Vlora, Albania \\ ${ }^{2}$ Department of Mathematics, Riphah International University, Faisalabad Campus, Satyana Road, Faisalabad, Pakistan \\ ${ }^{3}$ Comsats University Islamabad, Lahore Campus, Lahore, Pakistan \\ ${ }^{4}$ Virtual University of Pakistan, Lahore Campus, Lahore, Pakistan \\ ${ }^{5}$ Department of Mathematics, College of Arts and Sciences, Prince Sattam Bin Abdulaziz University, \\ Wadi Aldawaser 11991, Saudi Arabia \\ ${ }^{6}$ Department of Mathematics and General Sciences, Prince Sultan University, Riyadh 12345, Saudi Arabia \\ ${ }^{7}$ Department of Medical Research, China Medical University, Taichung 40402, Taiwan \\ ${ }^{8}$ Department of Computer Science and Information Engineering, Asia University, Taichung, Taiwan
}

Correspondence should be addressed to Thabet Abdeljawad; tabdeljawad@psu.edu.sa

Received 27 May 2020; Accepted 24 August 2020; Published 16 October 2020

Academic Editor: Basil K. Papadopoulos

Copyright ( 2020 Artion Kashuri et al. This is an open access article distributed under the Creative Commons Attribution License, which permits unrestricted use, distribution, and reproduction in any medium, provided the original work is properly cited.

\begin{abstract}
In this paper, the authors investigated the concept of $(s, m)$-exponential-type convex functions and their algebraic properties. New generalizations of Hermite-Hadamard-type inequality for the $(s, m)$-exponential-type convex function $\psi$ and for the products of two $(s, m)$-exponential-type convex functions $\psi$ and $\phi$ are proved. Many refinements of the $(\mathrm{H}-\mathrm{H})$ inequality via $(s, m)$-exponential-type convex are obtained. Finally, several new bounds for special means and new error estimates for the trapezoidal and midpoint formula are provided as well. The ideas and techniques of this paper may stimulate further research in different areas of pure and applied sciences.
\end{abstract}

\section{Introduction}

Theory of convexity also played a significant role in the development of theory of inequalities. Many famously known results in inequalities theory can be obtained using the convexity property of the functions. Hermite-Hadamard's double inequality is one of the most intensively studied results involving convex functions. This result provides us necessary and sufficient condition for a function to be convex. It is also known as classical equation of $\mathrm{H}-\mathrm{H}$ inequality.

The Hermite-Hadamard inequality assert that if a function $\psi: J \subset \Re \longrightarrow \Re$ is convex in $J$ for $a_{1}, a_{2} \in J$ and $a_{1}<a_{2}$, then

$$
\psi\left(\frac{a_{1}+a_{2}}{2}\right) \leq \frac{1}{a_{2}-a_{1}} \int_{a_{1}}^{a_{2}} \psi(\chi) d \chi \leq \frac{\psi\left(a_{1}\right)+\psi\left(a_{2}\right)}{2} .
$$

Interested readers can refer to [1-29].
Definition 1 (see [30]). A function $\psi:[0,+\infty) \longrightarrow \mathfrak{R}$ is said to be $s$-convex in the second sense for a real number $s \in(0,1]$ or $\psi$ belongs to the class of $K_{s}^{2}$, if

$$
\psi\left(\chi a_{1}+(1-\chi) a_{2}\right) \leq \chi^{s} \psi\left(a_{1}\right)+(1-\chi)^{s} \psi\left(a_{2}\right),
$$

holds for all $a_{1}, a_{2} \in[0,+\infty)$ and $\chi \in[0,1]$.

A $s$-convex function was introduced in Breckner's article in [30], and a number of properties and connections with $s$-convexity in the first sense are discussed in [10]. Usually, convexity means for $s$-convexity when $s=1$. Dragomir et al. proved a variant of Hadamard's inequality in [5], which holds for $s$-convex functions in the second sense. In the last decade, many mathematicians added the rich literature in the field of mathematical inequalities involving fractional calculus (see $[8,9,13,15,22,25,28,31]$ ).

Toader introduced the class of $m$-convex functions in [26]. 
Definition 2 (see [26]). A function $\psi:\left[0, a_{2}\right] \longrightarrow \mathfrak{R}, a_{2}>0$, is said to be $m$-convex, where $m \in(0,1]$, if

$$
\psi\left(\chi \theta_{1}+m(1-\chi) \theta_{2}\right) \leq \chi \psi\left(\theta_{1}\right)+m(1-\chi) \psi\left(\theta_{2}\right)
$$

holds $\forall \theta_{1}, \theta_{2} \in\left[0, a_{2}\right]$ and $\chi \in[0,1]$. Otherwise, $\psi$ is $m$-concave if $(-\psi)$ is $m$-convex.

In a recent paper, Eftekhari [6] defined the class of $(s, m)$-convex functions in the second sense as follows.

Definition 3. A function $\psi:[0,+\infty) \longrightarrow \mathfrak{R}$ is said to be $(s, m)$-convex for some fixed real numbers $s, m \in(0,1]$ if

$$
\psi\left(\chi a_{1}+m(1-\chi) a_{2}\right) \leq \chi^{s} \psi\left(a_{1}\right)+m(1-\chi)^{s} \psi\left(a_{2}\right)
$$

holds $\forall a_{1}, a_{2} \in[0,+\infty)$ and $\chi \in[0,1]$.

Fractional integral inequalities are useful to find the uniqueness of solutions for certain fractional partial differential equations (see $[19,32]$ ).

Let $\psi \in L\left[a_{1}, a_{2}\right]$. Then, Riemann-Liouville fractional integrals of order $\alpha>0$ with $a_{1} \geq 0$ are defined as follows:

$$
\begin{aligned}
& J_{a_{1}^{+}}^{\alpha} \psi(x)=\frac{1}{\Gamma(\alpha)} \int_{a_{1}}^{x}(x-\chi)^{\alpha-1} \psi(\chi) \mathrm{d} \chi, \quad x>a_{1}, \\
& J_{a_{2}^{-}}^{\alpha} \psi(x)=\frac{1}{\Gamma(\alpha)} \int_{x}^{a_{2}}(\chi-x)^{\alpha-1} \psi(\chi) \mathrm{d} \chi, \quad x<a_{2} .
\end{aligned}
$$

For further details, one may see $[14,16,18]$.

In $[7,17]$, there is given definition of $k$-fractional Riemann-Liouville integrals.

Let $\psi \in L\left[a_{1}, a_{2}\right]$. Then, $k$-fractional integrals of order $\alpha, k>0$ with $a_{1} \geq 0$ are defined as follows:

$$
\begin{aligned}
& { }^{k} J_{a_{1}^{+}}^{\alpha} \psi(x)=\frac{1}{k \Gamma_{k}(\alpha)} \int_{a_{1}}^{x}(x-\chi)^{\alpha / k-1} \psi(\chi) \mathrm{d} \chi, \quad x>a_{1}, \\
& { }^{k} J_{a_{2}^{-}}^{\alpha} \psi(x)=\frac{1}{k \Gamma_{k}(\alpha)} \int_{x}^{a_{2}}(\chi-x)^{\alpha / k-1} \psi(\chi) \mathrm{d} \chi, \quad x<a_{2},
\end{aligned}
$$

where $\Gamma_{k}(\alpha)$ is the $k$-gamma function defined as

$$
\Gamma_{k}(\alpha)=\int_{0}^{+\infty} \chi^{\alpha-1} e^{-\chi^{k} / k} \mathrm{~d} \chi
$$

We can notice that

$$
\begin{aligned}
& \Gamma_{k}(\alpha+k)=\alpha \Gamma_{k}(\alpha), \\
& { }^{1} J_{a_{1}^{+}}^{0} \psi(x)={ }^{1} J_{a_{2}^{-}}^{0} \psi(x)=\psi(x) .
\end{aligned}
$$

By choosing $k=1$, the above $k$-fractional integrals yield Riemann-Liouville integrals.

Also that, the incomplete gamma function $\gamma(\vartheta, \theta)$ is defined for $\vartheta>0$ and $\theta \geq 0$ by integral

$$
\gamma(\vartheta, \theta)=\int_{0}^{\theta} e^{-\mu} \mu^{\vartheta-1} \mathrm{~d} \mu .
$$

The gamma function $\Gamma(\vartheta)$ is defined for $\vartheta>0$ by integral

$$
\Gamma(\vartheta)=\int_{0}^{+\infty} e^{-\mu} \mu^{\vartheta-1} \mathrm{~d} \mu .
$$

Motivated by above results and literatures, we will give first in Section 2 the concept of $(s, m)$-exponential-type convex function, and we will study some of their algebraic properties. In Section 3, we will prove new generalizations of Hermite-Hadamard-type inequality for the $(s, m)$-exponential-type convex function $\psi$ and for the products of two $(s, m)$-exponential-type convex functions $\psi$ and $\phi$. In Section 4 , we will obtain some refinements of the $(\mathrm{H}-\mathrm{H})$ inequality for functions whose first derivative in absolute value at certain power is $(s, m)$-exponential-type convex. In Section 5, some new bounds for special means and error estimates for the trapezoidal and midpoint formula will be provided. In Section 6, a briefly conclusion will be given as well.

\section{Some Algebraic Properties of $(s, m)$-Exponential-Type Convex Functions}

In this section, we will introduce a new definition, called $(s, m)$-exponential-type convex function, and we will study some basic algebraic properties of it.

Definition 4. A nonnegative function $\psi: J \longrightarrow \Re$ is said $(s, m)$-exponential-type convex for some fixed $s, m \in(0,1]$ if

$$
\psi\left(\chi \theta_{1}+m(1-\chi) \theta_{2}\right) \leq\left(e^{s \chi}-1\right) \psi\left(\theta_{1}\right)+m\left(e^{(1-\chi) s}-1\right) \psi\left(\theta_{2}\right)
$$

holds for all $\theta_{1}, \theta_{2} \in J$ and $\chi \in[0,1]$.

Remark 1. For $m=s=1$, we get exponential- type convexity given by İşcan in [11].

Remark 2. ( $s, m)$-exponential-type convex functions for some fixed $m \in(0,1]$ and $s \in[\ln 2.5,1]$ have the range $[0,+\infty)$.

Proof. Let $\theta \in J$ be arbitrary for some fixed $m \in(0,1]$ and $s \in[\ln 2.5,1]$. Using the Definition 4 for $\chi=1$, we have

$$
\psi(\theta) \leq\left(e^{s}-1\right) \psi(\theta) \Longrightarrow\left(e^{s}-2\right) \psi(\theta) \geq 0 \Longrightarrow \psi(\theta) \geq 0 \text {. }
$$

Lemma 1. For all $\chi \in[0,1]$ and for some fixed $m \in(0,1]$ and $s \in[\ln 2.5,1]$, the following inequalities $\left(e^{s \chi}-1\right) \geq \chi^{s}$ and $\left(e^{(1-\chi) s}-1\right) \geq(1-\chi)^{s}$ hold.

Proof. The proof is evident.

Proposition 1. Every nonnegative $(s, m)$-convex function is $(s, m)$-exponential-type convex function for some fixed $m \in(0,1]$ and $s \in[\ln 2.5,1]$. 
Proof. By using Lemma 1, for some fixed $m \in(0,1]$ and $s \in[\ln 2.5,1]$, we have

$$
\begin{aligned}
& \psi\left(\chi \theta_{1}+m(1-\chi) \theta_{2}\right) \leq \chi^{s} \psi\left(\theta_{1}\right)+m(1-\chi)^{s} \psi\left(\theta_{2}\right) \\
& \leq\left(e^{s \chi}-1\right) \psi\left(\theta_{1}\right)+m\left(e^{(1-\chi) s}-1\right) \psi\left(\theta_{2}\right) .
\end{aligned}
$$

Theorem 1. Let $\psi, \phi:\left[a_{1}, a_{2}\right] \longrightarrow \mathfrak{R}$. If $\psi$ and $\phi$ are $(s, m)$-exponential-type convex functions for some fixed $s, m \in(0,1]$, then the following holds:

(1) $\psi+\phi$ is $(s, m)$-exponential-type convex function.

(2) For nonnegative real number $c, c \psi$ is $(s, m)$-exponential-type convex function.

Proof. By Definition 4, for some fixed $s, m \in(0,1]$, the proof is obvious.

Theorem 2. Let $\psi:\left[0, a_{2}\right] \longrightarrow J$ be $m$-convex function for $a_{2}>0$ and some fixed $m \in(0,1]$ and $\phi: J \longrightarrow \mathfrak{R}$ is nondecreasing and $(s, m)$-exponential-type convex function for some fixed $s, m \in(0,1]$. Then, for the same fixed numbers $s, m \in(0,1]$, the function $\phi^{\circ} \psi:\left[0, a_{2}\right] \longrightarrow \mathfrak{R}$ is $(s, m)$-exponential-type convex.

Proof. For all $\theta_{1}, \theta_{2} \in\left[0, a_{2}\right]$ and $\chi \in[0,1]$ and for some fixed numbers $s, m \in(0,1]$, we have

$$
\begin{aligned}
& \left(\phi^{\circ} \psi\right)\left(\chi \theta_{1}+m(1-\chi) \theta_{2}\right)=\phi\left(\psi\left(\chi \theta_{1}+m(1-\chi) \theta_{2}\right)\right) \\
& \leq \phi\left(\chi \psi\left(\theta_{1}\right)+m(1-\chi) \psi\left(\theta_{2}\right)\right) \\
& \leq\left(e^{s \chi}-1\right)\left(\phi^{\circ} \psi\right)\left(\theta_{1}\right)+m\left(e^{(1-\chi) s}-1\right)\left(\phi^{\circ} \psi\right)\left(\theta_{2}\right) .
\end{aligned}
$$

Theorem 3. Let $\psi_{i}:\left[a_{1}, a_{2}\right] \longrightarrow \mathfrak{R}$ be an arbitrary family of $(s, m)$-exponential-type convex functions for the same fixed $s, m \in(0,1]$ and let $\psi(\theta)=\sup _{i} \psi_{i}(\theta)$. If $A=\left\{\theta \in\left[a_{1}, a_{2}\right]\right.$ : $\psi(\theta)<+\infty\} \neq \varnothing$, then $A$ is an interval and $\psi$ is $(s, m)$-exponential-type convex function on $A$.

Proof. For all $\theta_{1}, \theta_{2} \in A$ and $\chi \in[0,1]$ and for the same fixed numbers $s, m \in(0,1]$, we have

$$
\begin{aligned}
& \psi\left(\chi \theta_{1}+m(1-\psi) \theta_{2}\right)=\sup _{i} \psi_{i}\left(\chi \theta_{1}+m(1-\chi) \theta_{2}\right) \\
& \leq \sup _{i}\left[\left(e^{s \chi}-1\right) \psi_{i}\left(\theta_{1}\right)+m\left(e^{(1-\chi) s}-1\right) \psi_{i}\left(\theta_{2}\right)\right] \\
& \leq\left(e^{s \chi}-1\right) \sup _{i} \psi_{i}\left(\theta_{1}\right)+m\left(e^{(1-\chi) s}-1\right) \sup _{i} \psi_{i}\left(\theta_{2}\right) \\
& =\left(e^{s \chi}-1\right) \psi\left(\theta_{1}\right)+m\left(e^{(1-\chi) s}-1\right) \psi\left(\theta_{2}\right)<+\infty .
\end{aligned}
$$

This means simultaneously that $A$ is an interval, and $\psi$ is $(s, m)$-exponential-type convex function on $A$.

Theorem 4. If the function $\psi:\left[a_{1}, a_{2}\right] \longrightarrow \mathfrak{R}$ is $(s, m)$-exponential-type convex for some fixed $s, m \in(0,1]$, then $\psi$ is bounded on $\left[a_{1}, m a_{2}\right]$.
Proof. Let $L=\max \left\{\psi\left(a_{1}\right), \psi\left(a_{2} / m\right)\right\}$ and $x \in\left[a_{1}, a_{2}\right]$ be an arbitrary point for some fixed $m \in(0,1]$. Then, there exists $\chi \in[0,1]$ such that $x=\chi a_{1}+(1-\chi) a_{2}$. Thus, since $e^{s \chi} \leq e^{s}$ and $e^{(1-\chi) s} \leq e^{s}$ for some fixed $s \in(0,1]$, we have

$$
\begin{aligned}
\psi(x)= & \psi\left(\chi a_{1}+(1-\chi) a_{2}\right) \leq\left(e^{s \chi}-1\right) \psi\left(a_{1}\right) \\
& +m\left(e^{(1-\chi) s}-1\right) \psi\left(\frac{a_{2}}{m}\right) \\
\leq & \left(e^{s}-1\right) L+m\left(e^{s}-1\right) L=(m+1) L\left(e^{s}-1\right)=M .
\end{aligned}
$$

We have shown that $\psi$ is bounded above from real number $M$. Interested reader can also prove the fact that $\psi$ is bounded below using the same idea as in Theorem 4 in [11].

\section{New Generalizations of $(\mathbf{H}-\mathbf{H})$ - Type Inequality}

In this section, we will establish some new generalizations of Hermite-Hadamard-type inequality for the $(s, m)$-exponential-type convex function $\psi$ and for the products of two $(s, m)$-exponential-type convex functions $\psi$ and $\phi$.

Theorem 5. Let $\psi:\left[a_{1}, m a_{2}\right] \longrightarrow \mathfrak{R}$ be $(s, m)$-exponentialtype convex function for some fixed $s, m \in(0,1]$ and $a_{1}<m a_{2}$. If $\psi \in L_{1}\left(\left[a_{1}, m a_{2}\right]\right)$, then

$$
\begin{aligned}
& \frac{1}{\left(e^{s / 2}-1\right)} \psi\left(\frac{a_{1}+m a_{2}}{2}\right) \leq \frac{1}{\left(m a_{2}-a_{1}\right)} \\
& \left\{\int_{a_{1}}^{m a_{2}} \psi(x) \mathrm{d} x+m \int_{a_{1} / m}^{a_{2}} \psi(x) \mathrm{d} x\right\} \\
& \leq\left(\frac{e^{s}-s-1}{s}\right)\left[\psi\left(a_{1}\right)+\psi\left(a_{2}\right)+m\left(\psi\left(\frac{a_{1}}{m^{2}}\right)+\psi\left(a_{2}\right)\right)\right] .
\end{aligned}
$$

Proof. Let denote, respectively,

$$
\theta_{1}=\chi a_{1}+m(1-\chi) a_{2}, \theta_{2}=(1-\chi) \frac{a_{1}}{m}+\chi a_{2}, \quad \forall \chi \in[0,1] .
$$

Using $(s, m)$-exponential-type convexity of $\psi$, we have

$$
\begin{aligned}
& \psi\left(\frac{a_{1}+m a_{2}}{2}\right)=\psi\left(\frac{\theta_{1}+m \theta_{2}}{2}\right) \\
& =\psi\left(\frac{\left[\chi a_{1}+m(1-\chi) a_{2}\right]+\left[(1-\chi) a_{1}+m \chi a_{2}\right]}{2}\right) \\
& \leq\left(e^{s / 2}-1\right)\left[\psi\left(\chi a_{1}+m(1-\chi) a_{2}\right)+\psi\left((1-\chi) a_{1}+m \chi a_{2}\right)\right] .
\end{aligned}
$$

Now, integrating on both sides in the last inequality with respect to $\chi$ over $[0,1]$, we get 


$$
\begin{aligned}
& \psi\left(\frac{a_{1}+m a_{2}}{2}\right) \leq\left(e^{s / 2}-1\right) \times\left[\int_{0}^{1} \psi\left(\chi a_{1}+m(1-\chi) a_{2}\right) \mathrm{d} \chi\right. \\
& \left.\quad+\int_{0}^{1} \psi\left((1-\chi) \frac{a_{1}}{m}+\chi a_{2}\right) \mathrm{d} \chi\right] \\
& =\frac{\left(e^{s / 2}-1\right)}{\left(m a_{2}-a_{1}\right)}\left\{\int_{a_{1}}^{m a_{2}} \psi(x) \mathrm{d} x+m \int_{a_{1} / m}^{a_{2}} \psi(x) \mathrm{d} x\right\},
\end{aligned}
$$

which completes the left-side inequality. For the right-side inequality, using $(s, m)$-exponential-type convexity of $\psi$, we obtain

$$
\begin{aligned}
& \frac{1}{\left(m a_{2}-a_{1}\right)}\left\{\int_{a_{1}}^{m a_{2}} \psi(x) \mathrm{d} x+m \int_{\frac{a_{1}}{m}}^{a_{2}} \psi(x) \mathrm{d} x\right\} \\
& =\int_{0}^{1} \psi\left(\chi a_{1}+m(1-\chi) a_{2}\right) \mathrm{d} \chi+\int_{0}^{1} \psi\left((1-\chi) \frac{a_{1}}{m}+\chi a_{2}\right) \mathrm{d} \chi \\
& \leq \int_{0}^{1}\left[\left(e^{s \chi}-1\right) \psi\left(a_{1}\right)+m\left(e^{(1-\chi) s}-1\right) \psi\left(a_{2}\right)\right] \mathrm{d} \chi \\
& +\int_{0}^{1}\left[\left(e^{s \chi}-1\right) \psi\left(a_{2}\right)+m\left(e^{(1-\chi) s}-1\right) \psi\left(\frac{a_{1}}{m^{2}}\right)\right] \mathrm{d} \chi \\
& =\left(\frac{e^{s}-s-1}{s}\right)\left[\psi\left(a_{1}\right)+\psi\left(a_{2}\right)+m\left(\psi\left(\frac{a_{1}}{m^{2}}\right)+\psi\left(a_{2}\right)\right)\right]
\end{aligned}
$$

which gives the right-side inequality.

Corollary 1. By taking $m=s=1$ in Theorem 5, we get (Theorem 5, [11]).

Theorem 6. Assume that $\psi, \phi:\left[a_{1}, m a_{2}\right] \longrightarrow \mathfrak{R}$ are, respectively, $\left(s_{1}, m\right)$ and $\left(s_{2}, m\right)$-exponential-type convex functions for the same fixed $m \in(0,1]$ and for some fixed $s_{1}, s_{2} \in(0,1]$, where $s_{1}<s_{2}$ and $a_{1}<m a_{2}$. If $\psi, \phi$ are synchronous functions and $\psi, \phi, \psi \phi \in L_{1}\left(\left[a_{1}, m a_{2}\right]\right)$, then

$$
\begin{aligned}
& \frac{1}{\left(m a_{2}-a_{1}\right)} \int_{a_{1}}^{m a_{2}} \psi(\theta) \mathrm{d} \theta \cdot \frac{1}{\left(m a_{2}-a_{1}\right)} \int_{a_{1}}^{m a_{2}} \phi(\theta) \mathrm{d} \theta \\
& \leq \frac{1}{\left(m a_{2}-a_{1}\right)} \int_{a_{1}}^{m a_{2}} \psi(\theta) \phi(\theta) \mathrm{d} \theta \\
& \leq A\left(s_{1}, s_{2}\right) M_{m}\left(a_{1}, a_{2}\right)+B\left(s_{1}, s_{2}\right) N\left(a_{1}, a_{2}\right),
\end{aligned}
$$

where

$$
\begin{aligned}
M_{m}\left(a_{1}, a_{2}\right) & =\psi\left(a_{1}\right) \phi\left(a_{1}\right)+m^{2} \psi\left(a_{2}\right) \phi\left(a_{2}\right), N\left(a_{1}, a_{2}\right) \\
& =\psi\left(a_{1}\right) \phi\left(a_{2}\right)+\psi\left(a_{2}\right) \phi\left(a_{1}\right) \\
A\left(s_{1}, s_{2}\right) & =\frac{e^{s_{1}+s_{2}}+s_{1}+s_{2}-1}{s_{1}+s_{2}}-\frac{s_{1}\left(e^{s_{2}}-1\right)+s_{2}\left(e^{s_{1}}-1\right)}{s_{1} s_{2}} \\
B\left(s_{1}, s_{2}\right) & =\frac{e^{s_{2}}-e^{s_{1}}}{s_{2}-s_{1}}-\frac{s_{1}\left(e^{s_{2}}-1\right)+s_{2}\left(e^{s_{1}}-1\right)}{s_{1} s_{2}}+1 .
\end{aligned}
$$

Proof. Let us denote $\theta=\chi a_{1}+m(1-\chi) a_{2}$ for all $\chi \in[0,1]$. Using the property of the $\left(s_{1}, m\right)$ and $\left(s_{2}, m\right)$-exponentialtype convex functions $\psi$ and $\phi$, respectively, we have

$$
\begin{aligned}
& \psi\left(\chi a_{1}+m(1-\chi) a_{2}\right) \leq\left(e^{s_{1} \chi}-1\right) \psi\left(a_{1}\right)+m\left(e^{(1-\chi) s_{1}}-1\right) \psi\left(a_{2}\right), \\
& \phi\left(\chi a_{1}+m(1-\chi) a_{2}\right) \leq\left(e^{s_{2} \chi}-1\right) g\left(a_{1}\right)+m\left(e^{(1-\chi) s_{2}}-1\right) \phi\left(a_{2}\right) .
\end{aligned}
$$

Multiplying above inequalities on both sides, we get

$$
\begin{aligned}
\psi & \left(\chi a_{1}+m(1-\chi) a_{2}\right) \phi\left(\chi a_{1}+m(1-\chi) a_{2}\right) \\
\leq & {\left[\left(e^{s_{1} \chi}-1\right) \psi\left(a_{1}\right)+m\left(e^{(1-\chi) s_{1}}-1\right) \psi\left(a_{2}\right)\right] } \\
& \times\left[\left(e^{s_{2} \chi}-1\right) g\left(a_{1}\right)+m\left(e^{(1-\chi) s_{2}}-1\right) \phi\left(a_{2}\right)\right] \\
= & \left(e^{s_{1} \chi}-1\right)\left(e^{s_{2} \chi}-1\right) \psi\left(a_{1}\right) \phi\left(a_{1}\right) \\
& +m\left[\left(e^{s_{1} \chi}-1\right)\left(e^{(1-\chi) s_{2}}-1\right) \psi\left(a_{1}\right) \phi a_{2}\right) \\
& \left.+\left(e^{s_{2} \chi}-1\right)\left(e^{(1-\chi) s_{1}}-1\right) \psi\left(a_{2}\right) \phi\left(a_{1}\right)\right] \\
& +m^{2}\left(e^{(1-\chi) s_{1}}-1\right)\left(e^{(1-\chi) s_{2}}-1\right) \psi\left(a_{2}\right) \phi\left(a_{2}\right) .
\end{aligned}
$$

Applying Chebyshev integral inequality (see [23]), we obtain

$$
\begin{aligned}
& \int_{0}^{1} \psi\left(\chi a_{1}+m(1-\chi) a_{2}\right) \phi\left(\chi a_{1}+m(1-\chi) a_{2}\right) \mathrm{d} \chi \\
& \geq \int_{0}^{1} \psi\left(\chi a_{1}+m(1-\chi) a_{2}\right) \mathrm{d} \chi \cdot \int_{0}^{1} \phi\left(\chi a_{1}+m(1-\chi) a_{2}\right) \mathrm{d} \chi
\end{aligned}
$$

Changing the variable of integration, we get

$$
\begin{aligned}
& \frac{1}{\left(m a_{2}-a_{1}\right)} \int_{a_{1}}^{m a_{2}} \psi(\theta) \phi(\theta) \mathrm{d} \theta \\
& \geq \frac{1}{\left(m a_{2}-a_{1}\right)} \int_{a_{1}}^{m a_{2}} \psi(\theta) \mathrm{d} \theta \cdot \frac{1}{\left(m a_{2}-a_{1}\right)} \int_{a_{1}}^{m a_{2}} \phi(\theta) \mathrm{d} \theta,
\end{aligned}
$$

which completes the left-side inequality. For the right-side inequality, integrating on both sides of the inequality (25) with respect to $\chi$ over $[0,1]$, we have 


$$
\begin{aligned}
& \frac{1}{\left(m a_{2}-a_{1}\right)} \int_{a_{1}}^{m a_{2}} \psi(\theta) \mathrm{d} \theta \cdot \frac{1}{\left(m a_{2}-a_{1}\right)} \int_{a_{1}}^{m a_{2}} \phi(\theta) \mathrm{d} \theta \\
& \leq\left[\int_{0}^{1}\left(e^{s_{1} \chi}-1\right)\left(e^{s_{2} \chi}-1\right) \mathrm{d} \chi\right] \psi\left(a_{1}\right) \phi\left(a_{1}\right) \\
& +m\left[\left(\int_{0}^{1}\left(e^{s_{1} \chi}-1\right)\left(e^{(1-\chi) s_{2}}-1\right) \mathrm{d} \chi\right) \psi\left(a_{1}\right) \phi\left(a_{2}\right)\right. \\
& \left.+\left(\int_{0}^{1}\left(e^{s_{2} \chi}-1\right)\left(e^{(1-\chi) s_{1}}-1\right) \mathrm{d} \chi\right) \psi\left(a_{2}\right) \phi\left(a_{1}\right)\right] \\
& +m^{2}\left[\int_{0}^{1}\left(e^{(1-\chi) s_{1}}-1\right)\left(e^{(1-\chi) s_{2}}-1\right) \mathrm{d} \chi\right] \psi\left(a_{2}\right) \phi\left(a_{2}\right) \\
& =A\left(s_{1}, s_{2}\right) M_{m}\left(a_{1}, a_{2}\right)+B\left(s_{1}, s_{2}\right) N\left(a_{1}, a_{2}\right),
\end{aligned}
$$

which give the right-side inequality.

\section{Refinements of (H-H)-Type Inequality for $k$ - Fractional Integral}

In this section, we will obtain some refinements of the $(\mathrm{H}-\mathrm{H})$ inequality via $(s, m)$-exponential-type convex functions.

Lemma 2. Suppose $0<w \leq 1$ and a mapping $\psi:\left[w a_{1}, a_{2}\right] \longrightarrow \mathfrak{R}$ is differentiable on $\left(w a_{1}, a_{2}\right)$ with $0<a_{1}<a_{2}$. If $\psi^{\prime} \in L_{1}\left[w a_{1}, a_{2}\right]$, then the following equality for $k$-fractional integral holds:

$$
\begin{aligned}
& \frac{\psi\left(w a_{1}\right)+\alpha / k \psi\left(a_{2}\right)}{\alpha / k+1}-\frac{\Gamma_{k}(\alpha+k)}{\left(a_{2}-w a_{1}\right)^{\alpha / k}} J_{a_{2}^{-}}^{\alpha} \psi\left(w a_{1}\right) \\
& =\left(\frac{a_{2}-w a_{1}}{\alpha / k+1}\right) \int_{0}^{1}\left[\left(\frac{\alpha}{k}+1\right) \chi^{\alpha / k}-1\right] \psi^{\prime}\left(w(1-\chi) a_{1}+\chi a_{2}\right) \mathrm{d} \chi
\end{aligned}
$$

where $\alpha, k>0$ and $\Gamma(\cdot)$ is the Euler Gamma function.

$$
\begin{aligned}
& \left(\frac{a_{2}-w a_{1}}{\alpha / k+1}\right) \int_{0}^{1}\left[\left(\frac{\alpha}{k}+1\right) \chi^{\alpha / k}-1\right] \psi^{\prime}\left(w(1-\chi) a_{1}+\chi a_{2}\right) \mathrm{d} \chi \\
& =\left(\frac{a_{2}-w a_{1}}{\alpha / k+1}\right)\left\{\int_{0}^{1}\left(\frac{\alpha}{k}+1\right) \chi^{\alpha / k} \psi^{\prime}\left(w(1-\chi) a_{1}+\chi a_{2}\right) \mathrm{d} \chi\right. \\
& \left.-\int_{0}^{1} \psi^{\prime}\left(w(1-\chi) a_{1}+\chi a_{2}\right) \mathrm{d} \chi\right\} \\
& =\left(\frac{a_{2}-w a_{1}}{\alpha / k+1}\right)\left[( \frac { \alpha } { k } + 1 ) \left\{\left.\frac{\chi^{\alpha / k} \psi\left(w(1-\chi) a_{1}+\chi a_{2}\right)}{a_{2}-w a_{1}}\right|_{0} ^{1}\right.\right. \\
& \left.-\int_{0}^{1} \frac{\psi\left(w(1-\chi) a_{1}+\chi a_{2}\right)}{a_{2}-w a_{1}} \frac{\alpha}{k} \chi^{\alpha / k-1} \mathrm{~d} \chi\right\} \\
& \left.-\left.\frac{\psi\left(w(1-\chi) a_{1}+\chi a_{2}\right)}{a_{2}-w a_{1}}\right|_{0} ^{1}\right] \\
& =\left(\frac{a_{2}-w a_{1}}{\alpha / k+1}\right)\left[\left(\frac{\alpha}{k}+1\right) \times\left\{\frac{\psi\left(a_{2}\right)}{a_{2}-w a_{1}}-\frac{\alpha}{k\left(a_{2}-w a_{1}\right)}\right.\right. \\
& \left.\left.\int_{0}^{1} \chi^{\alpha / k-1} \psi\left(w(1-\chi) a_{1}+\chi a_{2}\right) \mathrm{d} \chi\right\}\right] \\
& =\frac{\psi\left(w a_{1}\right)+\alpha / k \psi\left(a_{2}\right)}{\alpha / k+1}-\frac{\Gamma_{k}(\alpha+k)}{\left(a_{2}-w a_{1}\right)^{\alpha / k}} J_{a_{2}^{-}}^{\alpha} \psi\left(w a_{1}\right),
\end{aligned}
$$

which completes the proof.

Lemma 3. Suppose $0<w \leq 1$ and a mapping $\psi:\left[w a_{1}, a_{2}\right] \longrightarrow \mathfrak{R}$ is differentiable on $\left(w a_{1}, a_{2}\right)$ with $0<a_{1}<a_{2}$. If $\psi^{\prime} \in L_{1}\left[w a_{1}, a_{2}\right]$, then the following equality for $k$-fractional integral holds:

$$
\begin{aligned}
& \frac{\psi\left(w a_{1}\right)+\psi\left(a_{2}\right)}{w+1}-\frac{\Gamma_{k}(\alpha+k)}{(w+1)\left(a_{2}-w a_{1}\right)^{\alpha / k}} \\
& \left\{{ }^{k} J_{a_{1}^{+}}^{\alpha} \psi\left(a_{2}\right)+{ }^{k} J_{a_{2}^{-}}^{\alpha} \psi\left(w a_{1}\right)\right\} \\
& =\left(\frac{a_{2}-w a_{1}}{w+1}\right) \int_{0}^{1}\left[\chi^{\alpha / k}-(1-\chi)^{\alpha / k}\right] \psi^{\prime}\left(w(1-\chi) a_{1}+\chi a_{2}\right) \mathrm{d} \chi .
\end{aligned}
$$

Proof. Using the integrating by parts, we have 


$$
\begin{aligned}
& \left(\frac{a_{2}-w a_{1}}{w+1}\right) \int_{0}^{1}\left[\chi^{\alpha / k}-(1-\chi)^{\alpha / k}\right] \psi^{\prime}\left(w(1-\chi) a_{1}+\chi a_{2}\right) \mathrm{d} \chi \\
& =\left(\frac{a_{2}-w a_{1}}{w+1}\right)\left[\int_{0}^{1} \chi^{\alpha / k} \psi^{\prime}\left(w(1-\chi) a_{1}+\chi a_{2}\right) \mathrm{d} \chi\right. \\
& \left.\quad-\int_{0}^{1}(1-\chi)^{\alpha / k} \psi^{\prime}\left(w(1-\chi) a_{1}+\chi a_{2}\right) \mathrm{d} \chi\right] \\
& =\left(\frac{a_{2}-w a_{1}}{w+1}\right)\left[I_{1}-I_{2}\right],
\end{aligned}
$$

where

$$
\begin{aligned}
I_{1}= & \int_{0}^{1} \chi^{\alpha / k \psi^{\prime}\left(w(1-\chi) a_{1}+\chi a_{2}\right) \mathrm{d} \chi} \\
= & \left.\frac{\chi^{\alpha / k} \psi\left(w(1-\chi) a_{1}+\chi a_{2}\right)}{a_{2}-w a_{1}}\right|_{0} ^{1} \\
& -\int_{0}^{1} \frac{\psi\left(w(1-\chi) a_{1}+\chi a_{2}\right)}{a_{2}-w a_{1}} \frac{\alpha}{k} \chi^{\alpha / k-1} \mathrm{~d} \chi \\
= & \frac{\psi\left(a_{2}\right)}{a_{2}-w a_{1}}-\frac{\alpha}{k\left(a_{2}-w a_{1}\right)} \int_{0}^{1} \chi^{\alpha / k-1} \psi\left(w(1-\chi) a_{1}+\chi a_{2}\right) \mathrm{d} \chi \\
= & \frac{\psi\left(a_{2}\right)}{a_{2}-w a_{1}}-\frac{\Gamma_{k}(\alpha+k)}{\left(a_{2}-w a_{1}\right)^{\alpha / k+1}} J_{a_{2}^{-}}^{\alpha} \psi\left(w a_{1}\right), \\
I_{2}= & \int_{0}^{1}(1-\chi)^{\alpha / k} \psi^{\prime}\left(w(1-\chi) a_{1}+\chi a_{2}\right) \mathrm{d} \chi \\
= & \left.\frac{(1-\chi)^{\alpha / k} \psi\left(w(1-\chi) a_{1}+\chi a_{2}\right)}{a_{2}-w a_{1}}\right|_{0} ^{1} \\
& -\int_{0}^{1} \frac{\psi\left(w(1-\chi) a_{1}+\chi a_{2}\right)}{a_{2}-w a_{1}} \frac{\alpha}{k}(1-\chi)^{\alpha / k-1}(-1) \mathrm{d} \chi \\
= & -\frac{\psi\left(w a_{1}\right)}{a_{2}-w a_{1}}+\frac{\alpha}{k\left(a_{2}-w a_{1}\right)} \\
& \int_{0}^{1}(1-\chi)^{\alpha / k-1} \psi\left(w(1-\chi) a_{1}+\chi a_{2}\right) \mathrm{d} \chi \\
= & -\frac{\psi\left(w a_{1}\right)}{a_{2}-w a_{1}}+\frac{\Gamma_{k}(\alpha+k)}{\left(a_{2}-w a_{1}\right)^{\alpha / k+1}} J_{a_{1}^{\alpha} \psi}^{\alpha} \psi\left(a_{2}\right) . \\
&
\end{aligned}
$$

Combining equations (33) and (34) in (32) and multiplying by $a_{2}-w a_{1} / w+1$, we get (31), which completes the proof.

Theorem 7. Suppose $0<w \leq 1$ and a mapping $\psi:\left(0, a_{2} / m w\right] \longrightarrow \Re$ is differentiable on $\left(0, a_{2} / m w\right)$ with $0<a_{1}<a_{2}$. If $\left|\psi^{\prime}\right|^{q}$ is $(s, m)$-exponential-type convex on $\left(0, a_{2} / m w\right]$ for $q>1$ and $q^{-1}+p^{-1}=1$, then for some fixed $s, m \in(0,1]$, then the following inequality for $k$-fractional integral holds:

$$
\begin{aligned}
& \left|\frac{\psi\left(w a_{1}\right)+\alpha / k \psi\left(a_{2}\right)}{\alpha / k+1}-\frac{\Gamma_{k}(\alpha+k)}{\left(a_{2}-w a_{1}\right)^{\alpha / k}} J_{a_{2}^{-}}^{\alpha} \psi\left(w a_{1}\right)\right| \\
& \leq\left(\frac{a_{2}-w a_{1}}{\alpha / k+1}\right)\left[U_{1}(\alpha, k, p)+U_{2}(\alpha, k, p)\right]^{1 / p} \\
& \quad \times\left[\left(\frac{e^{s}-s-1}{s}\right)\left(\left|\psi^{\prime}\left(w a_{1}\right)\right|^{q}+m\left|\psi^{\prime}\left(\frac{a_{2}}{m}\right)\right|^{q}\right)\right]^{1 / q},
\end{aligned}
$$

where

$$
\begin{aligned}
& U_{1}(\alpha, k, p)=\int_{0}^{1 / \sqrt{[\alpha]}(\alpha / k+1)^{k}}\left(1-\left(\frac{\alpha}{k}+1\right) \chi^{\alpha / k}\right)^{p} \mathrm{~d} \chi, \\
& U_{2}(\alpha, k, p)=\int_{1 / \sqrt{[\alpha]}(\alpha / k+1)^{k}}^{1}\left(\left(\frac{\alpha}{k}+1\right) \chi^{\alpha / k}-1\right)^{p} \mathrm{~d} \chi .
\end{aligned}
$$

Proof. From Lemma 2, Hölder's inequality and $(s, m)$-exponential-type convexity of $\left|\psi^{\prime}\right|^{q}$, we have

$$
\begin{aligned}
&\left|\frac{\psi\left(w a_{1}\right)+\alpha / k \psi\left(a_{2}\right)}{\alpha / k+1}-\frac{\Gamma_{k}(\alpha+k)^{k}}{\left(a_{2}-w a_{1}\right)^{\alpha / k}} J_{a_{2}^{-}}^{\alpha} \psi\left(w a_{1}\right)\right| \\
& \leq\left(\frac{a_{2}-w a_{1}}{\alpha / k+1}\right) \int_{0}^{1}\left|\left(\frac{\alpha}{k}+1\right) \chi^{\alpha / k}-1\right|\left|\psi^{\prime}\left(w(1-\chi) a_{1}+\chi a_{2}\right)\right| \mathrm{d} \chi \\
& \leq\left(\frac{a_{2}-w a_{1}}{\alpha / k+1}\right)\left(\int_{0}^{1}\left|\left(\frac{\alpha}{k}+1\right) \chi^{\alpha / k}-1\right|^{p} \mathrm{~d} \chi\right)^{1 / p} \\
&\left(\int_{0}^{1}\left|\psi^{\prime}\left(w(1-\chi) a_{1}+\chi a_{2}\right)\right|^{q} \mathrm{~d} \chi\right)^{1 / q} \\
& \leq\left(\frac{a_{2}-w a_{1}}{\alpha / k+1}\right)\left(\int_{0}^{1}\left|\left(\frac{\alpha}{k}+1\right) \chi^{\alpha / k}-1\right|^{p} \mathrm{~d} \chi\right)^{1 / p} \\
& \times\left(\left.\int_{0}^{1}\left|\left(e^{(1-\chi) s}-1\right)\right| \psi^{\prime}\left(w a_{1}\right)\right|^{q}+m\left(e^{s \chi}-1\right)\left|\psi^{\prime}\left(\frac{a_{2}}{m}\right)\right|^{q} \mid \mathrm{d} \chi\right)^{1 / q} \\
&=\left(\frac{a_{2}-w a_{1}}{\alpha / k+1}\right)\left[U_{1}(\alpha, k, p)+U_{2}(\alpha, k, p)\right]^{1 / p} \\
& \times\left[\left(\frac{e^{s}-s-1}{s}\right)\left(\left|\psi^{\prime}\left(w a_{1}\right)\right|^{q}+m\left|\psi^{\prime}\left(\frac{a_{2}}{m}\right)\right|^{q}\right)\right]^{1 / q},
\end{aligned}
$$

which completes the proof.

Theorem 8. Suppose $0<w \leq 1$ and a mapping $\psi:\left(0, a_{2} / m w\right] \longrightarrow \Re$ is differentiable on $\left(0, a_{2} / m w\right)$ with $0<a_{1}<a_{2}$. If $\left|\psi^{\prime}\right|^{q}$ is $(s, m)$-exponential-type convex on $\left(0, a_{2} / m w\right]$ for $q \geq 1$, then for some fixed $s, m \in(0,1]$, then the following inequality for $k$-fractional integral holds: 


$$
\begin{aligned}
& \left|\frac{\psi\left(w a_{1}\right)+\alpha / k \psi\left(a_{2}\right)}{\alpha / k+1}-\frac{\Gamma_{k}(\alpha+k)}{\left(a_{2}-w a_{1}\right)^{\alpha / k}} J_{a_{2}^{-}}^{\alpha} \psi\left(w a_{1}\right)\right| \\
& \leq\left(\frac{a_{2}-w a_{1}}{\alpha / k+1}\right)\left(\frac{2 \alpha}{k(\alpha / k+1)^{k / \alpha+1}}\right)^{1-1 / q} \\
& \quad \times\left[| \psi ^ { \prime } ( w a _ { 1 } ) | ^ { q } \left\{-\frac{2 \alpha}{k(\alpha / k+1)^{k / \alpha+1}}-\frac{2}{s} e^{s\left(1-1 / \sqrt{[\alpha]}(\alpha / k+1)^{k}\right)}-\left(\frac{\alpha}{k}+1\right) \frac{e^{s}}{s^{\alpha / k}} \gamma\left(\frac{\alpha}{k}+1, \frac{1}{\sqrt{[\alpha](\alpha / k+1)^{k}}}\right)\right.\right. \\
& \left.\quad+\left(\frac{\alpha}{k}+1\right) e^{s}\left(s^{-\alpha / k}\right) \gamma_{\left(1-1 / \sqrt{[\alpha]}(\alpha / k+1)^{k}\right)}\left(\frac{\alpha}{k}+1, \frac{1}{\sqrt{[\alpha]}(\alpha / k+1)^{k}}\right)+\frac{1}{s}\right\} \\
& \quad+m\left|\psi^{\prime}\left(\frac{a_{2}}{m}\right)\right|\left\{\frac{2 e^{s / \sqrt{[\alpha]}(\alpha / k+1)^{k}}}{s}-\frac{2 \alpha}{k(\alpha / k+1)^{k / \alpha+1}}-\left(\frac{\alpha}{k}+1\right)\left(\frac{1}{(-s)^{\alpha / k}}\right) \gamma\left(\frac{\alpha}{k}+1, \frac{1}{\sqrt{[\alpha](\alpha / k+1)^{k}}}\right)\right. \\
& \left.\left.\quad+\left(\frac{\alpha}{k}+1\right)\left(\frac{1}{(-s)^{\alpha / k}}\right) \gamma_{1-1 / \sqrt{[\alpha]}(\alpha / k+1)^{k}}\left(\frac{\alpha}{k}+1, \frac{1}{\sqrt{[\alpha]}(\alpha / k+1)^{k}}\right)-\frac{e^{s}}{s}\right\}\right]^{1 / q} \cdot
\end{aligned}
$$

Proof. From Lemma 2, power mean inequality and $(s, m)$-exponential-type convexity of $\left|\psi^{\prime}\right|^{q}$, we have

$$
\begin{aligned}
\mid & \frac{\psi\left(w a_{1}\right)+\alpha / k \psi\left(a_{2}\right)}{\alpha / k+1}-\frac{\Gamma_{k}(\alpha+k)}{\left(a_{2}-w a_{1}\right)^{\alpha / k}} J_{a_{2}^{2}}^{\alpha} \psi\left(w a_{1}\right) \mid \\
\leq & \left(\frac{a_{2}-w a_{1}}{\alpha / k+1}\right) \int_{0}^{1}\left|\left(\frac{\alpha}{k}+1\right) \chi^{\alpha / k}-1\right|\left|\psi^{\prime}\left(w(1-\chi) a_{1}+\chi a_{2}\right)\right| \mathrm{d} \chi \\
\leq & \left(\frac{a_{2}-w a_{1}}{\alpha / k+1}\right)\left(\int_{0}^{1}\left|\left(\frac{\alpha}{k}+1\right) \chi^{\alpha / k}-1\right| \mathrm{d} \chi\right)^{1-1 / q} \times\left(\int_{0}^{1}\left|\left(\frac{\alpha}{k}+1\right) \chi^{\alpha / k}-1\right|\left|\psi^{\prime}\left(w(1-\chi) a_{1}+\chi a_{2}\right)\right|^{q} \mathrm{~d} \chi\right)^{1 / q} \\
\leq & \left(\frac{a_{2}-w a_{1}}{\alpha / k+1}\right)\left(\int_{0}^{1}\left|\left(\frac{\alpha}{k}+1\right) \chi^{\alpha / k}-1\right| \mathrm{d} \chi\right)^{1-1 / q} \times\left(\int_{0}^{1}\left|\left(\frac{\alpha}{k}+1\right) \chi^{\alpha / k}-1\right|\left[\left(e^{(1-\chi) s}-1\right)\left|\psi^{\prime}\left(w a_{1}\right)\right|^{q}+m\left(e^{s \chi}-1\right)\left|\psi^{\prime}\left(\frac{a_{2}}{m}\right)\right|^{q}\right] \mathrm{d} \chi\right)^{1 / q} \\
= & \left(\frac{a_{2}-w a_{1}}{\alpha / k+1}\right)\left(\frac{2 \alpha}{k(\alpha / k+1)^{k / \alpha+1}}\right)^{1-1 / q} \\
& \times\left[| \psi ^ { \prime } ( w a _ { 1 } ) | ^ { q } \left\{-\frac{2 \alpha}{k(\alpha / k+1)^{k / \alpha+1}}-\frac{2}{s} e^{s\left(1-1 / \sqrt{[\alpha]}(\alpha / k+1)^{k}\right)}-\left(\frac{\alpha}{k}+1\right) \frac{e^{s}}{s^{\alpha / k}} \gamma\left(\frac{\alpha}{k}+1, \frac{1}{\sqrt{[\alpha]}(\alpha / k+1)^{k}}\right)\right.\right. \\
& \left.+\left(\frac{\alpha}{k}+1\right) \frac{e^{s}}{s^{\alpha / k}} \gamma_{1-\frac{1}{\sqrt{[\alpha]}(\alpha / k+1)^{k}}}\left(\frac{\alpha}{k}+1, \frac{1}{\sqrt{[\alpha]}(\alpha / k+1)^{k}}\right)+\frac{1}{s}\right\} \\
& \left.\left.+\gamma\left(\frac{\alpha}{k}+1, \frac{1}{\sqrt{[\alpha]}(\alpha / k+1)^{k}}\right)+\left(\frac{\alpha}{k}+1\right)\left(\frac{1}{(-s)^{\alpha / k}}\right) \gamma_{1-1 / \sqrt{[\alpha]}(\alpha / k+1)^{k}}\left(\frac{\alpha}{k}+1, \frac{1}{\sqrt{[\alpha]}(\alpha / k+1)^{k}}\right)-\frac{e^{s}}{s}\right\}\right]^{1 / q}, \\
& +m\left|\psi^{\prime}\left(\frac{a_{2}}{m}\right)\right|\left\{\frac{2 e^{s / \sqrt{[\alpha]}(\alpha / k+1)^{k}}}{s}-\frac{2 \alpha}{k(\alpha / k+1)^{k / \alpha+1}}-\left(\frac{\alpha}{k}+1\right)\left(\frac{1}{(-s)^{\alpha / k}}\right)\right. \\
& \\
&
\end{aligned}
$$


which completes the proof.

Theorem 9. Suppose $0<w \leq 1$ and a mapping $\psi:\left(0, a_{2} / m\right] \longrightarrow \mathfrak{R}$ is differentiable on $\left(0, a_{2} / m\right)$ with $0<a_{1}<a_{2}$. If $\left|\psi^{\prime}\right|^{q}$ is $(s, m)$-exponential-type convex on $\left(0, a_{2} / m\right]$ for $q>1$ and $q^{-1}+p^{-1}=1$, then for some fixed $s, m \in(0,1]$, then the following inequality for $k$-fractional integral holds:

$$
\begin{aligned}
& \left|\frac{\psi\left(w a_{1}\right)+\psi\left(a_{2}\right)}{w+1}-\frac{\Gamma_{k}(\alpha+k)}{(w+1)\left(a_{2}-w a_{1}\right)}\left\{k J_{a_{1}^{+}}^{\alpha} \psi\left(a_{2}\right)+{ }^{k} J_{a_{2}^{-}}^{\alpha} \psi\left(w a_{1}\right)\right\}\right| \\
& \leq \frac{2\left(a_{2}-w a_{1}\right)}{w+1}\left(\frac{k}{\alpha p+k}\right)^{1 / p}\left[\left(\frac{e^{s}-s-1}{s}\right)\left(\left|\psi^{\prime}\left(w a_{1}\right)\right|^{q}+m\left|\psi^{\prime}\left(\frac{a_{2}}{m}\right)\right|^{q}\right)\right]^{1 / q} .
\end{aligned}
$$

Proof. From Lemma 3, Hölder's inequality and $(s, m)$-exponential-type convexity of $\left|\psi^{\prime}\right|^{q}$, we have

$$
\begin{aligned}
& \left|\frac{\psi\left(w a_{1}\right)+\psi\left(a_{2}\right)}{w+1}-\frac{\Gamma_{k}(\alpha+k)}{(w+1)\left(a_{2}-w a_{1}\right)^{\alpha / k}}\left\{J_{a_{1}^{+}}^{\alpha} \psi\left(a_{2}\right)+{ }^{k} J_{a_{2}^{-}}^{\alpha} \psi\left(w a_{1}\right)\right\}\right| \\
& \leq\left(\frac{a_{2}-w a_{1}}{w+1}\right) \int_{0}^{1}\left|\chi^{\alpha / k}-(1-\chi)^{\alpha / k}\right|\left|\psi^{\prime}\left(w(1-\chi) a_{1}+\chi a_{2}\right)\right| \mathrm{d} \chi \\
& \leq\left(\frac{a_{2}-w a_{1}}{w+1}\right)\left[\int_{0}^{1} \chi^{\alpha / k}\left|\psi^{\prime}\left(w(1-\chi) a_{1}+\chi a_{2}\right)\right| \mathrm{d} \chi+\int_{0}^{1}(1-\chi)^{\alpha / k}\left|\psi^{\prime}\left(w(1-\chi) a_{1}+\chi a_{2}\right)\right| \mathrm{d} \chi\right] \\
& \leq\left(\frac{a_{2}-w a_{1}}{w+1}\right)\left[\left(\int_{0}^{1} \chi^{\alpha / k p} d \chi\right)^{1 / p}\left(\int_{0}^{1}\left|\psi^{\prime}\left(w(1-\chi) a_{1}+\chi a_{2}\right)\right|^{q} \mathrm{~d} \chi\right)^{1 / q}+\left(\int_{0}^{1}(1-\chi)^{\alpha / k p} \mathrm{~d} \chi\right)^{1 / p}\left(\int_{0}^{1}\left|\psi^{\prime}\left(w(1-\chi) a_{1}+\chi a_{2}\right)\right|^{q} \mathrm{~d} \chi\right)^{1 / q}\right] \\
& \leq\left(\frac{a_{2}-w a_{1}}{w+1}\right)\left[\left(\int_{0}^{1} \chi^{\alpha / k p} \mathrm{~d} \chi\right)^{1 / p} \times\left(\int_{0}^{1}\left[\left(e^{(1-\chi) s}-1\right)\left|\psi^{\prime}\left(w a_{1}\right)\right|^{q}+m\left(e^{s \chi}-1\right)\left|\psi^{\prime}\left(\frac{a_{2}}{m}\right)\right|^{q}\right] \mathrm{d} \chi\right)^{1 / q}\right. \\
& \left.\quad+\left(\int_{0}^{1}(1-\chi)^{\alpha / k p} \mathrm{~d} \chi\right)^{1 / p} \times\left(\int_{0}^{1}\left[\left(e^{(1-\chi) s}-1\right)\left|\psi^{\prime}\left(w a_{1}\right)\right|^{q}+m\left(e^{s \chi}-1\right)\left|\psi^{\prime}\left(\frac{a_{2}}{m}\right)\right|^{q}\right] \mathrm{d} \chi\right)^{1 / q}\right] \\
& =\frac{2\left(a_{2}-w a_{1}\right)}{w+1}\left(\frac{k}{\alpha p+k}\right)^{1 / p}\left[\left(\frac{e^{s}-s-1}{s}\right)\left(\left|\psi^{\prime}\left(w a_{1}\right)\right|^{q}+m\left|\psi^{\prime}\left(\frac{a_{2}}{m}\right)\right|^{q}\right)\right]^{1 / q},
\end{aligned}
$$

which completes the proof.

Theorem 10. Suppose $0<w \leq 1$ and a mapping $\psi:\left(0, a_{2} / m\right] \longrightarrow \mathfrak{R}$ is differentiable on $\left(0, a_{2} / m\right)$ with $0<a_{1}<a_{2}$. If $\left|\psi^{\prime}\right|^{q}$ is $(s, m)$-exponential-type convex on $\left(0, a_{2} / m\right]$ for $q \geq 1$, then for some fixed $s, m \in(0,1]$, then the following inequality for $k$-fractional integral holds: 
Journal of Mathematics

9

$$
\begin{aligned}
& \left|\frac{\psi\left(w a_{1}\right)+\psi\left(a_{2}\right)}{w+1}-\frac{\Gamma_{k}(\alpha+k)}{(w+1)\left(a_{2}-w a_{1}\right)^{\alpha / k}}\left\{k J_{a_{1}^{+}}^{\alpha} \psi\left(a_{2}\right)+{ }^{k} J_{a_{2}^{-}}^{\alpha} \psi\left(w a_{1}\right)\right\}\right| \\
& \leq\left(\frac{a_{2}-w a_{1}}{w+1}\right)\left(\frac{k}{\alpha+k}\right)^{1-1 / q} \\
& \quad \times\left[\left\{\left|\psi^{\prime}\left(w a_{1}\right)\right|^{q}\left(\Gamma\left(\frac{\alpha}{k}+1\right)-\Gamma\left(\frac{\alpha}{k}+1, s\right) e^{s} s^{-\alpha / k-1}-\frac{1}{\alpha / k+1}\right)+m\left|\psi^{\prime}\left(\frac{a_{2}}{m}\right)\right|^{q}\left(\frac{\Gamma(\alpha / k+1,-s)-\Gamma(\alpha / k+1)}{(-s)^{\alpha / k} s}-\frac{1}{\alpha / k+1}\right)\right\}\right. \\
& +\left\{\left|\psi^{\prime}\left(w a_{1}\right)\right|^{q / q}\left((-1)^{\alpha / k-1} s^{-\alpha / k-1}\left(\Gamma\left(\frac{\alpha}{k}+1\right)-\Gamma\left(\frac{\alpha}{k}+1,-s\right)\right)-\frac{1}{\alpha / k+1}\right)\right. \\
& \left.\left.\quad+m\left|\psi^{\prime}\left(\frac{a_{2}}{m}\right)\right|^{q}\left(\frac{(\alpha / k+1) e^{s} k s^{-\alpha / k-1}(\Gamma(\alpha / k+1, s)-\Gamma(\alpha / k+1))}{k+\alpha}-\frac{1}{\alpha / k+1}\right)\right\}^{1 / q}\right]
\end{aligned}
$$

Proof. From Lemma 3, power mean inequality and $(s, m)$-exponential-type convexity of $\left|\psi^{\prime}\right|^{q}$, we have

$$
\begin{aligned}
& \left|\frac{\psi\left(w a_{1}\right)+\psi\left(a_{2}\right)}{w+1}-\frac{\Gamma_{k}(\alpha+k)}{(w+1)\left(a_{2}-w a_{1}\right)^{\alpha / k}}\left\{{ }^{k} J_{a_{1}^{+}}^{\alpha} \psi\left(a_{2}\right)+{ }^{k} J_{a_{2}^{-}}^{\alpha} \psi\left(w a_{1}\right)\right\}\right| \\
& \leq\left(\frac{a_{2}-w a_{1}}{w+1}\right) \int_{0}^{1}\left|\chi^{\alpha / k}-(1-\chi)^{\alpha / k}\right|\left|\psi^{\prime}\left(w(1-\chi) a_{1}+\chi a_{2}\right)\right| \mathrm{d} \chi \\
& \leq\left(\frac{a_{2}-w a_{1}}{w+1}\right) \times\left[\int_{0}^{1} \chi^{\alpha / k}\left|\psi^{\prime}\left(w(1-\chi) a_{1}+\chi a_{2}\right)\right| \mathrm{d} \chi+\int_{0}^{1}(1-\chi)^{\alpha / k}\left|\psi^{\prime}\left(w(1-\chi) a_{1}+\chi a_{2}\right)\right| \mathrm{d} \chi\right] \\
& \leq\left(\frac{a_{2}-w a_{1}}{w+1}\right)\left[\left(\int_{0}^{1} \chi^{\alpha / k} \mathrm{~d} \chi\right)^{1-1 / q}\left(\int_{0}^{1} \chi^{\alpha / k}\left|\psi^{\prime}\left(w(1-\chi) a_{1}+\chi a_{2}\right)\right|^{q} \mathrm{~d} \chi\right)^{1 / q}+\left(\int_{0}^{1}(1-\chi)^{\alpha / k} \mathrm{~d} \chi\right)^{1-1 / q}\right. \\
& \left.\left(\int_{0}^{1}(1-\chi)^{\alpha / k}\left|\psi^{\prime}\left(w(1-\chi) a_{1}+\chi a_{2}\right)\right|^{q} \mathrm{~d} \chi\right)^{1 / q}\right] \\
& \leq\left(\frac{a_{2}-w a_{1}}{w+1}\right)\left(\int_{0}^{1} \chi^{\alpha / k} \mathrm{~d} \chi\right)^{1-1 / q} \times\left[\left(\int_{0}^{1} \chi^{\alpha / k}\left[\left(e^{(1-\chi) s}-1\right)\left|\psi^{\prime}\left(w a_{1}\right)\right|^{q}+m\left(e^{s \chi}-1\right)\left|\psi^{\prime}\left(\frac{a_{2}}{m}\right)\right|^{q}\right] \mathrm{d} \chi\right)^{1 / q}\right. \\
& \left.+\left(\int_{0}^{1}(1-\chi)^{\alpha / k}\left[\left(e^{(1-\chi) s}-1\right)\left|\psi^{\prime}\left(w a_{1}\right)\right|^{q}+m\left(e^{s \chi}-1\right)\left|\psi^{\prime}\left(\frac{a_{2}}{m}\right)\right|^{q}\right] \mathrm{d} \chi\right)^{1 / q}\right] \\
& =\left(\frac{a_{2}-w a_{1}}{w+1}\right)\left(\frac{k}{\alpha+k}\right)^{1-1 / q} \\
& \times\left[\left\{\left|\psi^{\prime}\left(w a_{1}\right)\right|^{q}\left(\Gamma\left(\frac{\alpha}{k}+1\right)-\Gamma\left(\frac{\alpha}{k}+1, s\right) e^{s} s^{-\alpha / k-1}-\frac{1}{\alpha / k+1}\right)+m\left|\psi^{\prime}\left(\frac{a_{2}}{m}\right)\right|^{q}\left(\frac{\Gamma(\alpha / k+1,-s)-\Gamma(\alpha / k+1)}{(-s)^{\alpha / k} s}-\frac{1}{\alpha / k+1}\right)\right\}^{1 / q}\right. \\
& +\left\{\left|\psi^{\prime}\left(w a_{1}\right)\right|^{q}\left((-1)^{\alpha / k-1} s^{-\alpha / k-1}\left(\Gamma\left(\frac{\alpha}{k}+1\right)-\Gamma\left(\frac{\alpha}{k}+1,-s\right)\right)-\frac{1}{\alpha / k+1}\right)\right. \\
& \left.\left.+m\left|\psi^{\prime}\left(\frac{a_{2}}{m}\right)\right|^{q}\left(\frac{(\alpha / k+1) e^{s} k s^{-\alpha / k-1}(\Gamma(\alpha / k+1, s)-\Gamma(\alpha / k+1))}{k+\alpha}-\frac{1}{\alpha / k+1}\right)\right\}^{1 / q}\right] \text {, }
\end{aligned}
$$


which completes the proof.

\section{Applications}

Let consider the following two special means for different positive-real numbers $a_{1}<a_{2}$.

(1) The arithmetic mean:

$$
\mathscr{A}\left(a_{1}, a_{2}\right)=\frac{a_{1}+a_{2}}{2} .
$$

(2) The generalized log-mean:

$$
\mathscr{L}_{l}\left(a_{1}, a_{2}\right)=\left[\frac{a_{2}^{l+1}-a_{1}^{l+1}}{(l+1)\left(a_{2}-a_{1}\right)}\right]^{1 / l} ; l \in \Re \backslash\{-1,0\} .
$$

Dragomir et al. [5] have proved that, for $s \in(0,1)$, where $1 \leq l \leq 1 / s$, the function $f(x)=x^{l s}, x>0$ is $s$-convex function. Then, from Proposition 1 , it is also $s$-exponential convex function for some fixed $s \in[\ln 2.5,1)$.

Using Section 4, we have the following interesting results:

Proposition 2. Let $0<a_{1}<a_{2}, 0<w \leq 1$, and $q>1$ such that $p^{-1}+q^{-1}=1$. Then, for some fixed $s \in[\ln 2.5,1)$, where $1 \leq l \leq 1 / s$, we have

$$
\begin{aligned}
& \left|\mathscr{A}\left(\left(w a_{1}\right)^{l s}, a_{2}^{l s}\right)-\mathscr{L}_{l s}^{l s}\left(w a_{1}, a_{2}\right)\right| \leq \frac{l s\left(a_{2}-w a_{1}\right)}{\sqrt{[p]} 2} \\
& \quad \times\left(\frac{1}{p+1}\right)^{1 / p}\left(\frac{e^{s}-s-1}{s}\right)^{1 / q} \mathscr{A}^{1 / q}\left(\left(w a_{1}\right)^{(l s-1) q}, a_{2}^{(l s-1) q}\right) .
\end{aligned}
$$

Proof. Consider the $s$-exponential convex function $\psi(x)=x^{l s}, x>0$, and using Theorem 7 for $\alpha=1=k$, we obtain the required result.

Proposition 3. Let $0<a_{1}<a_{2}, 0<w \leq 1$ and $q \geq 1$. Then, for some fixed $s \in[\ln 2.5,1)$, where $1 \leq l \leq 1 /$ s, we get

$$
\begin{aligned}
\mid \mathscr{A} & \left(\left(w a_{1}\right)^{l s}, a_{2}^{l s}\right)-\mathscr{L}_{l s}^{l s}\left(w a_{1}, a_{2}\right) \mid \leq \frac{l s\left(a_{2}-w a_{1}\right)}{4^{(1-1 / q)}} \\
& \times\left(\frac{2(s-2) e^{s}+8 e^{s / 2}-s^{2}-2 s-4}{2 s^{2}}\right)^{1 / q} \\
& \mathscr{A}^{1 / q}\left(\left(w a_{1}\right)^{(l s-1) q}, a_{2}^{(l s-1) q}\right) .
\end{aligned}
$$

Proof. Consider the $s$-exponential convex function $\psi(x)=x^{l s}, x>0$, and using Theorem 8 for $\alpha=1=k$, the result is obvious.
Proposition 4. Let $0<a_{1}<a_{2}, 0<w \leq 1$ and $q>1$, such that $p^{-1}+q^{-1}=1$. Then, for some fixed $s \in[\ln 2.5,1)$, where $1 \leq l \leq 1 / s$, we obtain

$$
\begin{aligned}
& \left|\frac{2}{w+1}\left(\mathscr{A}\left(\left(w a_{1}\right)^{l s}, a_{2}^{l s}\right)-\mathscr{L}_{l s}^{l s}\left(w a_{1}, a_{2}\right)\right)\right| \\
& \leq \sqrt{[q]} 2 \frac{l s\left(a_{2}-w a_{1}\right)}{w+1} \times\left(\frac{1}{p+1}\right)^{1 / p}\left(\frac{e^{s}-s-1}{s}\right)^{1 / q} \\
& \mathscr{A}^{1 / q}\left(\left(w a_{1}\right)^{(l s-1) q}, a_{2}^{(l s-1) q}\right) .
\end{aligned}
$$

Proof. Consider the $s$-exponential convex function $\psi(x)=x^{l s}, x>0$, and using Theorem 9 for $\alpha=1=k$, the result is evident.

Proposition 5. Let $0<a_{1}<a_{2}, 0<w \leq 1$ and $q \geq 1$. Then, for some fixed $s \in[\ln 2.5,1)$, where $1 \leq l \leq 1 / s$, we have

$$
\begin{aligned}
& \left|\frac{2}{w+1}\left(\mathscr{A}\left(\left(w a_{1}\right)^{l s}, a_{2}^{l s}\right)-\mathscr{L}_{l s}^{l s}\left(w a_{1}, a_{2}\right)\right)\right| \leq \frac{l s\left(a_{2}-w a_{1}\right)}{2(w+1)} \\
& \quad \times\left(\frac{2(s-2) e^{s}+8 e^{s / 2}-s^{2}-2 s-4}{2 s^{2}}\right)^{1 / q} \\
& \quad \mathscr{A}^{1 / q}\left(\left(w a_{1}\right)^{(l s-1) q}, a_{2}^{(l s-1) q}\right) .
\end{aligned}
$$

Proof. Consider the $s$-exponential convex function $\psi(x)=x^{l s}, x>0$, and using Theorem 10 for $\alpha=1=k$, we obtain the required result.

At the end, let consider some applications of the integral inequalities obtained above, to find new bounds for the trapezoidal and midpoint formula.

For $a_{2}>0$, let $\mathcal{U}: 0=\chi_{0}<\chi_{1}<, \cdots,<\chi_{n-1}<\chi_{n}=a_{2}$ is a partition of $\left[0, a_{2}\right]$.

We denote

$$
\begin{aligned}
& \mathscr{T}(\mathcal{U}, \psi)=\sum_{i=0}^{n-1}\left(\frac{\psi\left(\chi_{i}\right)+\psi\left(\chi_{i+1}\right)}{2}\right) h_{i}, \\
& \int_{0}^{a_{2}} \psi(x) \mathrm{d} x=\mathscr{T}(\mathcal{U}, \psi)+\mathscr{R}(\mathcal{U}, \psi),
\end{aligned}
$$

where $\mathscr{R}(\mathcal{U}, \psi)$ is the remainder term and $h_{i}=\chi_{i+1}-\chi_{i}$ for $i=0,1,2, \ldots, n-1$.

Using above notations, we are in position to prove the following error estimations.

Proposition 6. Suppose a mapping $\psi:\left(0, a_{2}\right] \longrightarrow \mathfrak{R}$ is differentiable on $\left(0, a_{2}\right)$ with $a_{2}>0$. If $\left|\psi^{\prime}\right|^{q}$ is s-exponentialtype convex on $\left(0, a_{2}\right]$ for $q>1$ and $q^{-1}+p^{-1}=1$, then for some fixed $s \in(0,1]$, the remainder term satisfies the following error estimation: 


$$
\begin{gathered}
|\mathscr{R}(\mathcal{U}, \psi)| \leq \frac{1}{2}\left(\frac{1}{p+1}\right)^{1 / p}\left(\frac{e^{s}-s-1}{s}\right)^{1 / q} \\
\times \sum_{i=0}^{n-1} h_{i}^{2}\left[\left(\left|\psi^{\prime}\left(\chi_{i}\right)\right|^{q}+\left|\psi^{\prime}\left(\chi_{i+1}\right)\right|^{q}\right)\right]^{1 / q} .
\end{gathered}
$$

Proof. Using Theorem 7 on subinterval $\left[\chi_{i}, \chi_{i+1}\right]$ of closed interval $\left[0, a_{2}\right]$, for all $i=0,1,2, \ldots, n-1$ and $w=\alpha=k=m=1$, we get

$$
\begin{gathered}
\left|\left(\frac{\psi\left(\chi_{i}\right)+\psi\left(\chi_{i+1}\right)}{2}\right) h_{i}-\int_{\chi_{i}}^{\chi_{i+1}} \psi(x) \mathrm{d} x\right| \leq \frac{1}{2}\left(\frac{1}{p+1}\right)^{1 / p} \\
\left(\frac{e^{s}-s-1}{s}\right)^{1 / q} \times h_{i}^{2}\left[\left(\left|\psi^{\prime}\left(\chi_{i}\right)\right|^{q}+\left|\psi^{\prime}\left(\chi_{i+1}\right)\right|^{q}\right)\right]^{1 / q} .
\end{gathered}
$$

Summing inequality (52) over $i$ from 0 to $n-1$ and using the property of modulus, we obtain the desired inequality (51).

Proposition 7. Suppose a mapping $\psi:\left(0, a_{2}\right] \longrightarrow \mathfrak{R}$ is differentiable on $\left(0, a_{2}\right)$ with $a_{2}>0$. If $\left|\psi^{\prime}\right|^{q}$ is s-exponentialtype convex on $\left(0, a_{2}\right]$ for $q \geq 1$, then for some fixed $s \in(0,1]$, the remainder term satisfies the following error estimation:

$$
|\mathscr{R}(\mathcal{U}, \psi)| \leq\left(\frac{e^{s}-s-1}{2 s}\right)^{1 / q} \sum_{i=0}^{n-1} h_{i}^{2}\left[\left|\psi^{\prime}\left(\chi_{i}\right)\right|^{q}+\left|\psi^{\prime}\left(\chi_{i+1}\right)\right|^{q}\right]^{1 / q} .
$$

Proof. Apply the same technique as in Proposition 6 but using Theorem 8 .

\section{Conclusion}

In this article, the authors showed new generalizations of trapezium-type inequality for the new class of functions, the so-called $(s, m)$-exponential-type convex function $\psi$ and for the products of two $(s, m)$-exponential-type convex functions $\psi$ and $\phi$. We have obtained refinements of the $(\mathrm{H}-\mathrm{H})$ inequality for functions using $(s, m)$-exponential-type convex and founded new bounds for special means and for the error estimates for the trapezoidal and midpoint formula. We hope that current work will attract the attention of researchers working in mathematical analysis, fractional calculus, quantum calculus, postquantum calculus, and other related fields.

\section{Data Availability}

No data were used to support this study.

\section{Conflicts of Interest}

The authors declare that they have no conflicts of interest.

\section{References}

[1] T. Abdeljawad, S. Rashid, H. Khan, and Y. Chu, "On new fractional integral inequalities for $p$-convexity within interval valued functions," Advances in Difference Equations, vol. 330, 2020.

[2] T. Abdeljawad, S. Rashid, Z. Hammouch, and Y. Chu, "Some new local fractional inequalities associated with generalized $(s-m)$-convex functions and applications," Advances in Difference Equations, vol. 406, p. 27, 2020.

[3] M. Alomari, M. Darus, and U. S. Kirmaci, "Refinements of Hadamard-type inequalities for quasi-convex functions with applications to trapezoidal formula and to special means," Computers \& Mathematics with Applications, vol. 59, no. 1, pp. 225-232, 2010.

[4] F. Chen and S. Wu, "Several complementary inequalities to inequalities of Hermite-Hadamard type for $s$-convex functions," Journal of Nonlinear Sciences and Applications, vol. 9, no. 2, pp. 705-716, 2016.

[5] S. S. Dragomir and S. Fitzpatrik, "The Hadamard's inequality for $s$-convex functions in the second sense," Demonstratio Mathematica, vol. 32, no. 4, pp. 687-696, 1999.

[6] N. Eftekhari, "Some remarks on ( $s-m)$-convexity in the second sense," Journal of Mathematical Inequalities, vol. 8, no. 3, pp. 489-495, 2014.

[7] G. Farid, A. U. Rehman, and M. Zahra, "On Hadamard inequalities for $k$-fractional integrals," Nonlinear Functional Analysis and Applications, vol. 21, no. 3, pp. 463-478, 2016.

[8] G. Farid, U. N. Katugampola, and M. Usman, "Ostrowski type fractional integral inequalities for $S$-Godunova-Levin functions via katugampola fractional integrals," OMS, vol. 1, pp. 85-96, 2017.

[9] S. Guo and Y. Chu, "Fractional Hadamard and FejerHadamard inequaities associated with exponentially $(s-m)$ convex functions," Journal of Function Spaces, vol. 2020, p. 10, Article ID 2410385, 2020.

[10] H. Hudzik and L. Maligranda, "Some remarks on s-convex functions," Aequationes Mathematicae, vol. 48, no. 1, pp. 100-111, 1994.

[11] M. Kadakal and İ. İşcan, "Exponential type convexity and some related inequalities," Journal of Inequalities and Applications, vol. 2020, no. 1, pp. 1-9, 2020.

[12] A. Kashuri and R. Liko, "Some new hermite-hadamard type inequalities and their applications," Studia Scientiarum Mathematicarum Hungarica, vol. 56, no. 1, pp. 103-142, 2019.

[13] H. Ge-JiLe, S. Rashid, S. Rashid, M. Aslam Noor, A. Suhail, and Y.-M. Chu, "Some unified bounds for exponentially tgsconvex functions governed by conformable fractional operators," AIMS Mathematics, vol. 5, no. 6, pp. 6108-6123, 2020.

[14] R. Gorenflo and F. Mainardi, Fractional Calculus: Integral and Differential Equations of Fractional Order, Springer Verlag, Belin, Germany, 1997.

[15] H. Kalsoom, M. Idrees, D. Baleanu, and Y. Chu, "New estimates of $q 1 q 2$-Ostrowski-type inequalities with a class of npolynomial prevexity of function," Journal of Function Spaces, vol. 2020, p. 13, Article ID 3720798, 2020.

[16] K. Miller and B. Ross, An Introduction to the Fractional Calculus and Fractional Differential Equations, John Wiley \& Sons, New York, NY, USA, 1993.

[17] S. Mubeen and G. M. Habibullah, "k-Fractional integrals and applications," International Journal of Contemporary Mathematical Sciences, vol. 7, pp. 89-94, 2012.

[18] I. Podlubni, Fractional Differential Equations, Academic Press, San Diego, CA, USA, 1999. 
[19] A. A. Kilbas, H. M. Srivastava, and J. J. Trujillo, Theory and Applications of Fractional Differential Equations, Elsevier, Amsterdam, Netherlands, 2006.

[20] P. O. Mohammed, M. Z. Sarikaya, and D. Baleanu, "On the generalized hermite-hadamard inequalities via the tempered fractional integrals," Symmetry, vol. 12, no. 4, p. 595, 2020.

[21] O. Omotoyinbo and A. Mogbodemu, "Some new Hermite-Hadamard integral inequalities for convex functions," International Journal of Scientific Innovations, vol. 12, pp. 112, 2014

[22] H. Qi, M. Yussouf, and Y. Mehmood, "Fractional integral versions of Hermite-Hadamard type inequality for generalized exponentially convexity," AIMS Mathematics, vol. 5, no. 6, pp. 630-6042, 2020.

[23] A. Rafiq, N. A. Mir, and F. Ahmad, "Weighted ČebyševOstrowski type inequalities," Applied Mathematics and Mechanics, vol. 28, no. 7, pp. 901-906, 2007.

[24] E. Set, M. A. Noor, M. U. Awan, and A. Gözpinar, "Generalized Hermite-Hadamard type inequalities involving fractional integral operators," Journal of Inequalities and Applications, vol. 17, pp. 1-10, 2017.

[25] M. Sun and Y. Chu, "Inequalities for the generalized weighted mean values of $g$-convex functions with applications," Revista de la Real Academia de Ciencias Exactas, Físicas y Naturales. Serie A. Matemáticas, RACSAM, vol. 114, no. 4, 2020.

[26] G. Toader, Some Generalizations of the Convexity, Universityof Cluj-Napoca, in Proceedings of the Colloquium on Approximationand Optimization, pp. 329-338, Cluj-Napoca, Romania, 1985.

[27] B. Y. Xi and F. Qi, "Some integral inequalities of Hermite-Hadamard type for convex functions with applications to means," Journal of Function Spaces, vol. 114, Article ID 980438, 2012.

[28] X. Yang, G. Farid, W. Nazeer, M. Yussouf, Y. Chu, and C. Dong, "Fractional generalized Hadamard and FejerHadamard inequalities for $m$-convex function," AIMS Mathematcs, vol. 5, no. 6, pp. 6325-6340, 2020.

[29] X. M. Zhang, Y. M. Chu, and X. H. Zhang, "The Hermite-Hadamard type inequality of GA-convex functions and its applications," Journal of Inequalities and Applications, vol. 5, Article ID 507560, 2010.

[30] W. W. Breckner, "Stetigkeitsaussagen füreine Klasse verallgemeinerter konvexer funktionen in topologischen linearen Raumen," Publications de l'Institut Mathématique, vol. 23, pp. 13-20, 1978.

[31] S. I. Butt, "On caputo fractional derivatives via exponential ( $s-$ $m$ )-convex functions," Engineering and Applied Science Letters, vol. 3, no. 2, pp. 32-39, 2020.

[32] G. A. Anastassiou, Advanced Inequalities, World Scientifc, Singapore, 2011. 